\title{
Global aphasia and the language of thought ${ }^{\&}$
}

\author{
(Afasia global y el lenguaje del pensamiento)
}

\author{
Fred ADAMs* \\ University of Delaware
}

\begin{abstract}
Jerry Fodor's arguments for a language of thought (LOT) are largely theoretical. Is there any empirical evidence that supports the existence of LOT? There is. Research on Global Aphasia supports the existence of LOT. In this paper, I discuss this evidence and why it supports Fodor's theory that there is a language of thought.
\end{abstract}

KEYWORDS: Jerry Fodor, Rosemary Varley, language of thought, global aphasia, theory of mind, artificial grammar, Bliss symbols, dissociation of systems.

RESUMEN: Los argumentos de Fodor a favor de un lenguaje del pensamiento (LOT) son en gran parte teóricos. ¿Hay alguna evidencia empirica que apoye la existencia de LOT? La investigación sobre afasia global apoya la existencia de LOT. En este artículo, discuto esta evidencia y por qué apoya la teoría de Fodor de que hay un lenguaje del pensamiento.

PALABRAS CLAVE: Jerry Fodor, Rosemary Varley, lenguaje del pensamiento, afasia global, teoría de la mente, gramática artificial, simbolos Bliss, disociación de sistemas

\& I am extremely grateful to Rosemary Varley for generously answering many e-mailed questions about her work. During my research on this topic I have been helped by the following undergraduate students interested in undergraduate research at the University of Delaware: Saumya Sharan, Robyn Lachow, Jared Delffer, and Nate Fulham. Versions of this paper were presented at the Atiner Conference in Athens in May of 2018 and at the Morris Colloquium on the Self and its Realizations, UC-Boulder, June 16-18. I am grateful to the participants for feedback and to two anonymous referees for excellent suggestions and help revising.

* Correspondence to: Fred Adams. University of Delaware, Newark, Delaware (19716 USA) - fa@udel.edu

How to cite: Adams, Fred. (2020). «Global aphasia and the language of thought»; Theoria. An International Journal for Theory, History and Foundations of Science, 35(1), 9-27. (https://doi.org/10.1387/theoria.20430).

Received: 30 November, 2018; Final version: 3 July, 2019.

ISSN 0495-4548 - eISSN 2171-679X / (C) 2020 UPV/EHU 


\section{Introduction}

In a series of important works (Fodor, 1975, 1987, 2008), Jerry Fodor argued for the existence of a brain code, a mentalese, a language of thought (which has come to be known as LOT). Fodor mounted persuasive reasons why LOT is not a natural language. These include but are not limited to the following:

1. There are nonverbal organisms that think;

2. There are preverbal children that think;

3. Language learning requires hypothesis formation (i.e., thinking);

4. One cannot learn a language unless you already know one-which is innate (to avoid regress).

An additional part of the basis for this hypothesis is the fact that thought is structured. So support came from the parallel between the structure of spoken and written languages and the structure of thought itself. Spoken and written languages have a compositional structure to both their syntax and semantics. There is a productivity that can be explained in terms of the rules for construction. Similarly, thought itself seems to mirror the same structure and to be explainable by the same computational principles. ${ }^{1}$

Part of the basis for this hypothesis is provided by the kind of mental processes that are required for language learning. Fodor puts it this way:

...perceptual processing (perception of any kind but especially of language) presupposes a representational system rich enough to distinguish between sets of physical properties of sentence types, and being able to distinguish, phonological, acoustic, morphological, and syntactic properties of sentence tokens. (Fodor, 1975, 51)

Hence, much of the basis of the argument for thought having a language-like structure was an inference to the best explanation style of reasoning. That is: learning a language requires a set of mental operations that allow the above kinds of linguistic processing. What is the best explanation of the nature of thought that would explain the above list of thought's properties? It is that it involves language-like units with compositional syntax and semantics, viz. an innate language of thought or LOT. That is, there must be a medium for the computational processes of language learning. Hence, a list of the kinds of properties of thought that a compositional LOT would help explain include, but is not limited to the following:

1. Productivity (in principle infinite competence) (Fodor, 1987).

2. Systematicity (if one can believe $\mathrm{aRb}$, then one can believe bRa) (Fodor, 1987).

3. Recursivity (thoughts have a repeatable, nestable, compositional structure) (Fodor, 1975, 1987).

4. Ability to represent both actual and non-actual states of affairs (Fodor, 1975, 1987).

1 Of course, Fodor's general argument for the Representational Theory of Mind (RTM) also formed much of the basis for his defense of LOT. Not to be overlooked was the ability of LOT and RTM to solve «Frege's Puzzle» (Fodor, 2008). On this last point, see also (Adams \& Fuller, 1992, and Adams, Fuller, \& Stecker, 1993). 
So we can arrive at the conclusion via abductive reasoning for LOT with Fodor's own words:

\footnotetext{
We are back to our old point that psychological processes are typically computational and computation presupposes a medium for representing the structures over which the computational operations are defined. (Fodor, 1975, 51)
}

\section{Additional empirical support for $L O T$}

While Fodor (1975) included some evidence from psycholinguistics, most philosophers and linguists recognize his arguments for LOT as theoretical arguments. Indeed, not being an experimentalist by nature, it is hard to know what else Fodor might have appealed to in coming up with, articulating, and defending his arguments for LOT. However, not long ago I was introduced to the work of Rosemary Varley and her colleagues at University College London. They have had an ongoing research project studying subjects with what is know as Global Aphasia. Varley and her colleagues have come to believe that there are two systems in the brain that can dissociate: the language system and the cognitive system. Over the course of many interesting experiments, she and her colleagues have accumulated an impressive set of data to support that there is this dissociation in these subjects. In the remainder of this paper I will present the findings of some of her research (and related research on Global Aphasia). I will examine the strength of the defense of her thesis of dissociation, and I will address the matter of whether the work of Varley, her colleagues, and other researchers (Glass, A.V., Gazzaninga, M., \& Premack, D. 1973) on global aphasia offers empirical support for the existence of LOT. ${ }^{2}$

\section{What is global aphasia?}

Global aphasia is not just one thing. It is a family of deficits that vary with the amount of damage to language areas of the brain--damage that can be caused by lesions, stroke and various other neural insults.

Global aphasia is also distinct from other aphasias, such as Wernicke's aphasia (which causes impaired comprehension but not difficulty with word production, even semantically or syntactically garbled ones). It is also different than Broca's aphasia where comprehension is not impaired but subjects lack ability to produce language fluently. Global aphasia acts like a combination of these other two aphasias. Thus it is a much more severe form of aphasia, affecting both comprehension and production.

Symptoms of global aphasia include difficulty comprehending and producing verbs (Varley \& Willems, 2010). Sometimes symptoms include radical impairment of grammati-

2 Varley seems to me to be quite philosophically savvy and knowledgeable about philosophy. Still I don't remember one place in her research papers where she mentions «LOT.» Nonetheless, I think it is natural to assume that she knows that if she is right in her conclusion about the dissociation of the language system and the cognitive system, she is, in effect, supplying support for the possibility--indeed plausibility of LOT. 
cal and lexical capacity. Subjects typically have difficulty with word comprehension and word finding. Broca's aphasia is characterized by simplified syntactic structures in speech and writing and parallel difficulties in deriving semantic/thematic information from sentence structures. Subjects with global aphasia typically have difficulty making grammatical judgments about sentences and production (they may simply stringing together a sequence of nouns, rather than produce subject-predicate form) (Varley \& Siegal, 2000). As an example, each of three men with global aphasia (SA, SO and PR) was unable to understand the difference between sentences such as 'The man chased the dog/The dog chased the man' (Varley 2014). The subjects are at chance attempting to match sentences with pictures depicting the related actions.

Causes of aphasia include stroke, lesions to neural areas of the language system of the brain such as Broca's area and Wernicke's. There is often damage in prefrontal cortical language areas and superior temporal gyrus and the tissues between those regions. Patients typically have lesions within left hemisphere perisylvian cortex and associated subcortical structures such as the thalamus (Varley, 2014). Subjects with global aphasia often have an impairment of peripheral performance mechanisms involved in language, such as auditory perception (Zimmerer \& Varley, 2010).

So damage to language-mediating regions of the cortex and associated sub-cortical structures result in little or no ability to understand or construct sentences in any modality of language use (spoken or written). In addition to loss of grammatical ability there can also be substantial impairment of lexical knowledge. The mechanisms involved in relaying linguistic information to form a central competence are impaired in global aphasia (Siegal \& Varley, 2006).

\section{Agrammaticism}

Varley refers to the linguistic deficits associated with global aphasia as "agrammaticism." Here is an example of how she uses this term as applied to one subject $S$. A.

In summary, S.A. displayed a severe grammatical impairment across input and output processing modalities in sentence parsing (grammaticality judgments) and in sentence and verb comprehension. The term 'agrammatism' is used to refer to a spectrum of grammatical disorders, and the complete loss of grammatical ability is very rare. Individuals described as agrammatic normally have residual primitive grammatical capacity that enables them to understand and construct simple language propositions that would enable some grammatical support of thinking. In contrast, S.A.'s impairment was profound. He showed no evidence of an ability to formulate propositions in speech or writing. He was not able to make judgments as to whether a sentence is grammatical, or to match sentences to pictures, or to identify the meaning of verbs. (Varley \& Siegal, 2000, 724)

The deficits termed "agrammaticism" are severe. The patients described as agrammatic are unique in that they perform at chance on comprehensions of canonical active sentences of the type the boy kissed the girl/ the girl kissed the boy (Varley, 2014). Global aphasia profiles include lack of sensitivity to word order information, and therefore, incorrect performance on active sentences (Varley, 2014). Such subjects cannot understand cases where meaning cannot be extracted solely from the semantic content of the words alone (Zim- 
merer, Cowell, \& Varley 2010). For such subjects production is more greatly impaired compared to comprehension (for example, subject PR had low production scores but higher lexical comprehension scores) (Zimmerer, Cowell, \& Varley 2010).

\section{Subjects with agrammaticism have no significant cognitive deficit}

Based in part upon a study with subject SA (a retired police officer who has severe damage to left temporal language areas), Varley claims that agrammaticism does not hinder cognitive capacity. Varley and Siegal (2000) gave SA a battery of tests for grammatical competence. SA's performance on these tests were barely above chance. On this basis they claim he satisfies their criterion for being "agrammatical."3

Then they tested SA on two other cognitive tasks. The first was causal reasoning and the second was theory of mind (ToM) tasks. Varley- describes the causal reasoning task:

Causal reasoning test S.A. was presented with a picture card that depicted an event on the left-hand side (for example, a car crashed into a tree). On the right-hand side there were three pictures (the target, for example an alcoholic drink, and two semantic associates, for example a helicopter and an axe). S.A. pointed to indicate the likely cause of the event. In three training trials, the cue 'Why?' was printed on the card and the task explained to the participant. On the 15 test items, no further instructions were given. (Varley \& Segal, 2000, 726)

SA scored in the $84^{\text {th }}$ percentile of correct responses for Wechsler Adult Intelligence Scale (WAIS) picture arrangement tests. In this test:

The standard WAIS procedure was used. A series of line drawings were placed in front of the participant, who was asked to re-arrange the cards so that these told a sensible story (for example, a simple item involves three pictures displaying the stages in constructing a house). Responses were timed and scored for accuracy and speed. (Varley \& Segal, 2000, 726)

They also tested SA on theory of mind (ToM) reasoning. SA was shown a container containing unexpected items. For example, a pillbox containing buttons. He was then asked what another subject would "think" is in the container. Or he was asked what "really" was in the container. On the theory of mind tasks, although SA had some difficulty, he scored well above chance.

Based upon these results Varley \& Siegal (2000) draw the very strong conclusion that grammatical competence and cognitive competence can dissociate. Varley doesn't claim that cognition and grammatical ability completely dissociate (life long), but that in an adult

3 The method of determining that the subject is at «chance» level is as follows: a reversible sentence comprehension. Participant points at a picture that represents the interaction of two protagonists. The distractor picture shows the same protagonists in reverse agent - action - patient roles. There is both an auditory sentence-picture match, and written sentence-picture match. Included are equal numbers of sentences in active and passive voice, to rule out the strategy of assuming the first noun is always the agent. Hence, in cases where there is a picture of a man killing a lion and one of a lion killing a man, subjects are at chance matching "the man killed the lion" to the right picture. 
mind with significant insult to the language areas, these two competencies can come apart. They claim:

\begin{abstract}
Therefore, the pattern of responses revealed in this case of severe agrammatic aphasia attests to a dissociation between grammar and cognition and to the modular nature of mature brain functioning. Grammar may play a vital role in configuring cognitive processes, but once these processes have been established, cognition can operate without grammar. (Varley \& Siegel, 2000, 726)
\end{abstract}

This would be a very strong conclusion to draw based upon one subject, but as we shall see, she has access to several other subjects and other tests and data that she calls upon to support this strong claim.

Varley, Siegel, \& Want (2001) examined another subject MR with global aphasia and with what they've defined as agrammaticism. The damage to MR's brain resulted from a left cerebrial vascular accident with damage to lenticular striate cortex, basal ganlia and internal capsule and internal cortical damage to the perisylvian area. This resulted in grammatical deficits. He was at chance differentiating sentences such «The man killed the lion» vs. «the lion killed the man.» MR's speech consisted of fragments of stereotypic forms and little ability to construct novel language propositions.

Theory of Mind (ToM): since this topic comes up several times below, I briefly introduce the topic here. ${ }^{4}$ The label "theory of mind" (ToM) in the cognitive sciences has come to refer to the general capacity to attribute mental states to self and others (Goldman, 2012). This is the kind of capacity thought to be absent in individuals with autism (BaronCohen, 1995). It is also a capacity associated with the false-belief paradigm (Baron-Cohen, Leslie, \& Frith, 1985), wherein observers are able to attribute false beliefs to subjects. In a typical situation, a subject is shown an object in location A and then the subject leaves the room. The object is moved to location B. Then observers are asked, "Where will the subject look for the object when re-entering the room?" Will the subject look at location A (because he falsely believes) it is still at location A? Or will the subject believe it is at location $\mathrm{B}$ (where it has been moved). It is said that if the observer suggests that the subject believes the object is still at location $\mathrm{A}$, the observer has theory of mind. The observer attributes a false belief to the subject. Curiously, some children prior to age 4 will attribute to the subject the belief that the object is now at location B (even though not seeing the object being moved to location $B$ ). They attribute to the subject the thought that the object is "really" at location B, rather than the thought that the subject will mistakenly "think" it is at location A.

Despite his grammatical deficits, MR is at the $64^{\text {th }}$ percentile on causal reasoning and the WAIS test. And MR scored 5/5 on ToM (theory of mind) tasks involving distinguishing what a subject "thought" versus what was "really" the case.

On this basis Varley et. al. conclude that despite severe grammatical deficit MR's linguistic impairments do not preclude MR's ToM reasoning. They take this to show, as with

4 The explanation of how theory of mind works splits into two camps, the so-called «theory-theory» and the «simulation» account. The former actually attributes a theory-like structure to the subject who understands mental states and how they work. On the simulation account, subjects are said said to use the mental states to simulate the mental states of another, without actually building an internal theory. For more see (Adams, 2001). 
SA, a dissociation between grammatical ability and cognitive reasoning such as strong performance on ToM reasoning.

To attain answers from MR they described the method used as follows:

\footnotetext{
"The subject was then asked the belief question "what does the boy think he has caught?" or a reality question "what has the boy caught really?" Belief questions were prompted by a card depicting a character with a thought bubble. And reality questions were accompanied by a character beside a solid frame."
}

Varley (2014) describes in more detail than in earlier papers how subjects were trained to be able to respond to ToM questions. First, they trained on the "think/really" distinction. So, for example, SA was presented with a bag of balls and asked to guess how many balls the bag contained. As he guessed a flash card with a thought bubble with the word "think" was displayed. Later, he counted the ball actually in the bag in the presence of a second flash card with the word "really" showing. He then gave a "reality" answer about how many balls were really in the bag. That supposedly gave him an understanding of the difference between "think" and "really" and a way to express it (in relation to the flash cards).

Varley (2014) describes SA's performance as "not perfect," but that he was able to infer the knowledge states of others in the ToM tasks, even when the beliefs of subjects were false. As in a typical ToM task, SA was asked what a subject would "think" was in a container versus what was "really" in the container. We can assume the questions were facilitated by using the flash cards upon which SA was trained. SA's performance was well above chance. The conclusion Varley et. al. wish to draw is that SA's cognitive ToM performance was not mediated by language because of his agrammaticism.

In another type of experiment, Willems, RM., Benn, Y., Hagoort, P., Toni, I., \& Varley RA. (2011) and Varley (2014) describe the performance of subject with global aphasia on a communicative intentions game. There is a $3 \times 3$ grid with two tokens on the grid. One token is controlled by the sender and the other by the receiver. The receiver has to guess the intentions of the sender. The receiver does this by observing the type of token and its placement on the grid by the sender. Successful performance was the receiver interpreting the communicative intentions behind the movement of the sender's token, and subsequently positioning their token in its correct location and orientation.

The sender knows the final target location for both sender's and receiver's tokens and has to communicate this to the receiver. The sender has to develop strategies to communicate target positions to the receiver and receiver has to understand the communicative strategies being used by the sender.

The results in successful communication for subject ST in sender role was $80 \%$ and in receiver role $79 \%$. The desired conclusion is that the cognitive means by which the subjects with global aphasia achieved these results were not linguistic due to their agrammaticism.

\section{Some recursive-style reasoning without language}

PR was an English-speaking 62 year old man who suffered a stroke 7 years earlier. He had a large lesion in perisylvian language area. He also had damage to the inferior frontal gyrus, unsula and underlying deep white matter. Superior temporal, supramarginal and angular 
gyri were lesioned. Damage extended to the inferior border of the intraparietal sulcus. He could produce some high frequency words, but his word comprehension was severely impaired. Meaningful communication was only possible with an abundance of hand gestures, drawings and other intermediaries.

Investigating the cognitive capacities of subject PR, Varley, R, Klessinger, N., Romanowski, C. \& Segal, M (2005) discovered that PR could successfully compute mathematical equations that involved some recursive-style cognitive ability. For example, he could solve $50-((4+7) \times 4)]$.

In a further study, three subjects SA, SO, PR also displayed the ability to perform mathematical calculations requiring sensitivity to syntactic nesting of operations despite being "agrammatical" by Varley's definition. These subjects were able to correctly differentiate the mathematical operations $7-2$ vs. 2-7 and 20/5 vs. 5/20 and the more complex embedded equation $(5 \times(6+2))$ (Varley, 2014).

In considering whether this ability was due to some re-organization in the brain of aphasic subjects, they looked at fMRI data from these subjects and non-aphasic subjects during calculation. In aphasics, they saw no evidence of wide-scale reorganization. And in non-aphasics they saw evidence that some neural calculation is taking place not in language areas but in right hemisphere (Benn et al. 2012, 2013).

In addition, PR could also solve non-linguistic theory of mind (ToM) tasks. This finding is consistent with Varley's overall view that linguistic competence and cognitive competence can dissociate. This would also be consistent with the view of Pinker and Jackendoff (2005), who maintain that there is a general recursive cognitive capacity not dependent upon natural language.

However, Varley, R., Klessinger, N., Romanowski, C. \& Siegal, M. (2005) cannot draw the strong conclusion that PR's performance only supports the dissociation hypothesis. For they acknowledge that it is possible that linguistic capacity for recursion is the "mother of recursion," and that PR's earlier linguistic development and linguistic capacity was necessary for his current cognitive recursive capacities. So while PR's performance is consistent with the view that cognitive recursion and linguistic recursion can come apart, it is not conclusive evidence for that view. However, the fact that fMRI evidence reveals activation in non-language areas in non-aphasics is at least some additional evidence that language is not the "mother of recursion" (Varley, 2014).

\section{Failure to learn artificial grammar}

It turns out that PR is not able to learn the recursive rules for artificial grammar.

In one experiment researchers tried to teach subjects with Global Aphasia an artificial grammar that required grasping a kind of recursion. The rule was that a string was well formed if it included $\mathrm{A} / \mathrm{B}$ or a recursive function of the same: $\mathrm{A} / \mathrm{B}, \mathrm{AA} / \mathrm{BB}, \mathrm{AAA} / \mathrm{BBB}$, and so on (Hauser, Chomsky \& Fitch, 2002). And while he was above chance at rejecting some of the ungrammatical cases of the artificial grammar, he likely was using other, nonrecursive strategies in order to do so. He may have simply matched the numbers of strings of As and Bs in the artificial grammar tree by simply counting. This strategy would allow his answers to be co-extensive with the correct answers without actually having learned the grammatical rules of the artificial grammar. 
It may seem surprising that subjects do poorly on the learning of artificial grammar if their cognitive system supports recursion. However, there are several factors at play. First, the stimuli used were auditory. In several cases the damage to the language system also affected auditory systems. This may have impacted performance. Second, had stimuli been pictorial, subjects might have done better. Instead of $\mathrm{A} / \mathrm{B}, \mathrm{AA} / \mathrm{BB}, \mathrm{AAA} / \mathrm{BBB}$, they might have used pictures. In place of 'A's and 'B's they might have used pictures of dogs and cats. Third, if the subjects truly are attempting to treat the symbols as words and their linguistic system frankly is "shot," then it is not surprising at all that they cannot plug the inputs into a linguistic system and learn an artificial grammar. Lastly, as we shall see below, other forms of stimuli do seem to induce cognitive capacity that should remain if there is indeed a separate cognitive system of LOT. Indeed, when they switched stimuli to visual shapes and colors, Zimmerer, Cowell, and Varley (2014) did find some improvement in ability to learn artificial grammar.

Furthermore, the fact that subjects with global aphasia have significant ToM skills, suggests that these skills are not based solely upon language. Of course, Varley et. al. similarly may wonder that since these subjects previously had full linguistic capacity, developmentally language may have played a role in their ToM skills after acquiring global aphasia. That is, if language were the "mother of recursion," perhaps it was also the mother of ToM. The ToM skills of subjects with global aphasia may have required linguistic skills sometime in the past. Still, the fact that pre-linguistic infants have ToM skills, does not support the view that language is necessary for this cognitive capacity.

\section{Infant ToM}

There is at least some evidence in favor of the dissociation hypothesis in data from infants who apparently have some ToM skills at the age of 18 months (Baillargeon, Scott $\& \mathrm{He}, 2010)$. These infants look longer when a subject re-enters a room and looks for an object where it actually is rather than where it was when the subject left the room. The argument made on the basis of the infant looking paradigm is that the infant expects the subject to look where he falsely will believe the object to still be not look where it actually is.

Since the infants are pre-verbal, it is not fully developed language skills that explain their looking behavior. To the extent that these experiments demonstrate some ToM reasoning among infants, that behavior cannot be explained by their use of language. Of course, if Varley et. al. allow the possibility that "language is the mother of recursion," they may also have to allow the possibility that an innate universal grammar makes it possible for pre-verbal infants to exercise some ToM reasoning of the sort displayed in the infantlooking paradigm. However, in Varley's favor is the fact that unactualized capacities are less likely to explain actual looking behavior.

Of course there are other possible explanations of the differential looking times of infants in the ToM experiments. They could simply be doing some statistical modeling of human behavior without attributing the causes of that behavior to hidden causes such as beliefs. But to the extent that the infant-looking paradigm is accepted as displaying ToM reasoning, then it offers at least some support for the idea that they are doing this without actualized language skills. 


\section{What conclusions does Varley draw from her data?}

Based upon the above discussion of results, we can summarize the conclusions Varley and colleagues draw from their studies of subjects with Global Aphasia. Varley).

First, subjects with Global Aphasia have agrammaticism (as operationally defined by

Second, despite their agrammaticism experiments conducted by Varley et. al. support each of the following claims:

- subjects with Global Aphasia score in normal ranges on causal reasoning tasks and theory of mind (ToM) tasks;

- subjects with Global Aphasia performed in normal ranges on cognitive tasks requiring the ability to play a game of communicative intentions (as either an intention "sender" or "receiver");

— subjects with Global Aphasia have recursive reasoning ability (such as required to solve mathematical problems following associate rules and nesting within equations).

Finally, Varley et. al. draw the conclusion that the language system and the cognitive system are "autonomous" systems-at least in these subjects (Varley, 2014). They can function together in normal adult humans who have full language capacity. But they can dissociate in humans with global aphasia. All of her studies are designed to provide evidence in support of the view that there are two separate systems-the natural language system and the cognitive system. Her data suggests that these two systems can operate independently and do in persons with global aphasia.

\section{Does the data support the conclusions Varley et. al. draw from studies of subjects with global aphasia?}

One Issue that needs to be addressed in relation to Varley's data is the performance/competence distinction and whether it can explain why subjects that she calls "agrammatical" still have high cognitive ability. The difference between performance and competence in language is that a person may be able to understand language that the person cannot produce. For example, human infants can often understand sentences that their parents utter, but that the infants themselves cannot produce at that age.

Another example would be someone who learned a second language, but doesn't use it regularly. This person may be able to understand someone who addresses them in that second language, but the person may no longer be able to respond in the second language, because he/she didn't continue to actively use the second language.

When Varley and colleagues solicit data from subjects with global aphasia, they have to communicate with the subjects. Most answers they get from them are answers to questions in English. Whether it is theory of mind questions (ToM), mathematical operation questions, or communicative intentions interactions, the subjects have to understand the questions or prompts that are presented to them in English. How are they able to answer intelligently if they don't understand the questions? And if they do understand the questions, doesn't that indicate a certain level of linguistic competence with English? 
I posed this question to Varley via personal communication. Her answer was that, in the end, the subjects understood what they were being asked, but to get them to understand required much non-linguistic communication. Sometimes it required gestures. Sometimes it required drawings and other informal means of communication to get them to understand what they were being asked. I took her response to indicate that if the subjects genuinely had linguistic competence, the experimenters wouldn't have needed all the other tools with which to communicate.

A lingering worry is that despite the extra effort and tools required, when the subjects finally understood what they being asked, their understanding might be in the form of a linguistic representation of either the question or the task at hand. If the subjects are using linguistic representations to understand the questions or tasks, however difficult, however many extra kinds of effort and tools are involved, the experimental results would still not show that the subjects with global aphasia were using a separate cognitive system.

At the same time, in the defense of Varley and here colleagues, if the subjects who are judged to «agrammatical» even with extra effort, gestures, pictures etc. still cannot distinguish «the man killed the lion,» from «the lion killed the man,» something serious is going on at the level of linguistic representation.

Given that not being able to distinguish agent/patient interchanges in language is a center-piece in Varley's operational definition of «agrammatical,» she will count these subjects as lacking grammatical competence. But one may be uncertain whether this operational test demonstrates complete lack of linguistic competence. By analogy, we wouldn't say of a person with dyslexia that he/she «couldn't see.» We'd say that despite being able to see their visual system has a glitch. It visually reverses word order in some cases. So, by analogy, it may be possible that subjects with global aphasia have enough linguistic competence to, with much help and extra effort and tools, be able to understand questions and instructions in English. However, their linguistic system (due to neural insult in the linguistic areas of the brain), has a glitch and they cannot accurately distinguish agent/patient pairs linguistically.

The next worry is that although Varley and colleagues have access to subjects with global aphasia, the actual number of subjects tested is still quite small indeed. We understand the number of subjects available is not the same as running experiments with undergraduates at universities, where subjects are plentiful. So it is understandable why she has a small number of subjects (less than 10).

It is true that the evidence she obtained from her subjects tends to support the hypothesis that, while «agrammatical,» subjects had little other cognitive deficit. Of course, we don't know what would be found with a larger sample size.

However, the brain is a large and complex organ. Each of her subjects had damage to language areas of the brain (there were insults to the same general areas) but we don't know exactly how similar the damage was across subjects. So while they were all «agrammatical» by her tests, this might have been for quite different reasons across subjects. So the interaction between language and cognition could have varied significantly for each subject. One just doesn't know.

It is true that it is easier to find disconfirming evidence than confirming with small sample sizes (and Varley and colleagues are testing for disconfirming evidence-disconfirming that language competence is necessary for all cognitive competence). To confirm 
that all ravens are black, one must look at a lot of ravens. But to disconfirm, one need find only one white raven. Varley and colleagues were looking for white ravens, and that helps with the matter of small sample size.

Since there is a small sample size, it will be important whether other researchers studying subjects with aphasia are finding similar or dissimilar results.

\section{Support from other researchers}

Glass, Gazzaniga, \& Premack, 1973 found supporting data that is consistent with the research of Varley, et. al. They tested subjects with global aphasia on ability to learn artificial languages and discovered:

Despite gross deficits in natural language, these patients were able to learn an artificial language system using cut-out paper symbols for words.... and despite massive language loss globally aphasic patients retain a rich conceptual system and at least some capacity for symbolization and primitive linguistic functions. (Glass, Gazzaniga, \& Premack, 1973, 95)

They note that right hemisphere has semantic knowledge, but no syntactic knowledge. How do they know this? By studying split-brain subjects. In fact, Gazzaniga became very well known for his work in this area. They note that damage to language areas may leave pre-linguistic cognitive areas "intact." So they wanted to investigate the capacity for symbolization in patients with global aphasia. Their goal was to see if subjects could learn an artificial language system that Premack formerly used to study primates.

They had access to seven patients, all right-handed, all with global aphasia. They had severe language deficit, no language expressive capacity, one or two word limits, automatisms, phonemic jargon in specific situations, and could not spontaneously write or copy written words. Subjects had limited linguistic comprehension-and what little they did have was erratic and unreliable. They possessed limited recognition for three letter high frequency words.

Six subjects age 59-84 were otherwise motivated, bright, and eager to participate in the experiment and training. All were at least 3 months post stroke. All were given a pre-test on 60 word/non-word pairs. Two patients could not do this and their data was excluded. Of the four who remained: two made two errors on the pre-test. So, at best, they could identify these three-letter, high frequency single words.

Included in the pre-test, there was a "picture association test" matching pictures with words and the average correct response was at $54 \%$. After initial trials, on subsequent trials the patients were only at $59 \%$ recognition for words.

So their language skills were compromised by the damage to their language areas.

Some could distinguish words from non-sense syllables (but didn't know what words meant). This led to speculation that the subjects did this by retrieving a structural (picturelike) template for words (vs. non-words, for which there would be no template).

Furthermore, subjects failed to distinguish CVC (consonant-vowel-consonant) potential words from CCC (consonant-consonant-consonant) nonsense non-potential word pairs. None of the subjects could discriminate words by class: Noun, adjective, verb. However, given cut-out letters to spell words for associated pictures, 4 were able to spell words, 2 
were unable to spell words, 2 had semantic knowledge of words, but 2 did not even though they could spell the words (using the pictorial cut-outs).

None displayed knowledge of "singulars" vs. "plural” nouns. None displayed knowledge of agreement of nouns and verbs. None displayed knowledge of "three word sentences"-even with picture as visual aids.

Consistent with Varley's "agrammaticism," findings these subjects could not discriminate "A hits B," vs. "B hits A." What is more, none could comprehend or produce simple sentences. What is more, I think the findings of Glass, Gazzaniga, \& Premack are consistent with there being a lack of linguistic competence among these subjects.

Subjects were aware of surroundings. They understood appropriate vs. inappropriate uses of objects. They recognized pictures, displayed appropriate facial gestures for situations pictured. They were able to correctly sort pictures into piles: Animal/plant, animate/ inanimate, male/female, edible/non-edible plant, child/adult. So they had basic concepts and could understand the contrary categories for those concepts.

During the training period, cut outs of symbols of varying size and color were used. These could be arranged left to right to make grammatically correct sentences. Words were permanent visual symbols. At first the experimenter makes the words from the cut outs, the subjects merely use them. Sentences could be as small as one word. Each new word is taught as the only new word added to a prior string. The Format:

\footnotetext{
A same A

A different $B$

Gradually pictures are replaced with word symbols and queried. A?A A?B Or Or _ same B? same A?
}

The subjects seemed to catch on more quickly if material was familiar. One subject was a "card shark" and did better if symbols and words were related to playing cards. Another was a carpenter and did better with objects and words related to tools. Reward for success was as simple as a social signal of the experimenter being pleased, but sometimes food or candy was also supplied.

Subjects first learned on samples that were always correct. Then gradually there was a question symbol introduced "is A the same as A?" Is "A different from B?" and there were possibilities of errors. Finally they were given A/A or A/B and allowed to supply "same" or "different" themselves.

They first were trained on objects: a fork and a spoon. Then after they got things right, they were tested for transfer to non-test objects: pencil, eraser, etc. All subjects learned some variations of the cases. The average length of time required to learn was one month, but they learned more quickly after first successes when learning a new trial. And one patient could still perform the learned cases 4 months after the initial learning.

Even "negation" was introduced. "A not same B." Or "A not different B."

5 of the 7 subjects picked this up and picked up the "transfer" test to new non-training object pairs. Lexicons increased: they learned a few nouns, verbs, and persons names, learned by placing object 1 next to object 2 and placing the correct noun, verb, or name in between.

So they are learning to cobble together strings of cut out words and pictures (where the pictures were taking on the cognitive role of symbols) and they are putting them together 
in the logical form of subject-predicate sentences. Since their purely linguistic capacity to do this with language areas of the brain had been compromised, they were discovering a "work-around." They could learn to string together pictorial symbols for simple sentences such as "Andrea stir water." Other examples of symbols learned include: "Andrea," "John," "Tang," "water," "stir," "drink," "pour."

They became able to produce and comprehend three word sentences at $80 \%$ accuracy. All but 2 learned negation. All but 2 learned names. All but 1 learned simple verbs.

The experimenters were able to eliminate the possibility that this was simple $S$ - R learning. Subjects could comprehend and express very simple declarative sentences by this method, and their use of the symbols followed syntactic rules.

Words (syntax) can be stored separately from their semantic correlates.

In some cases, patients could make word/non-word discriminations even when they didn't know the meanings of the words (semantic content). In some cases they could discriminate CVC words from nonsense words-and to do so they must have used orthographic representations. So it seems that words were in part are stored as visuo-verbal units.

In some cases they could spell words for which they had no semantic comprehension (similar results occur in split-brain subjects). Subjects may have manipulated the cut-outs until they matched a visuo-verbal unit. The fact that patients often paid little or no attention to the pictures supports this view.

One patient spelled "god" with satisfaction though the picture was of a dog.

The authors surmised that it is possible that there are cognitive processes in both hemispheres. These patients succeeded at sophisticated conceptual sorting. And they don't suffer cognitive deficit in proportion to linguistic deficit. These are just the sorts of findings reported by Varley and colleagues.

Consistent with the research of Glass, Gazzaniga, \& Premack (1973) is the work of Garrett \& Beukelman (1995). Garrett \& Beukelman (1995) demonstrated subjects with global aphasia can improve communication by gesturing to pre-written sentences to convey thoughts. But this seems to be clearly a case of lack of linguistic performance, but does not close the door on the possibility of residual linguistic competence. So their study doesn't conclude that there is cognition completely independent of language.

However, Johannsen-Horbach, et al. (1985) used a set of "Blissymbols" (Bliss, 1965) to communicate with a subject with global aphasia. They discovered improved communication via these symbols that are more pictorial and iconic than words of a natural language. This study demonstrates different results than that of Garrett \& Beukelman (1995) because the communication is not via competence with natural language sentences. Since the Blissymbols are more pictorial and not words, this may help to support Varley's position that there are two separate systems at work (a language system and a non-linguistic cognitive system).

Blissymbols are a visual symbol system of pictograms and ideograms. In total, they comprise several hundred basic symbols, each representing a concept. The symbols can be put together and when composed can generate new symbols that represent new concepts.

Blissymbolics as a language has certain advantages in comparison to spoken or written language. Utterances can be produced by pointing or drawing and can be perceived by visuospatial analysis (very similar to the symbol system devised by Glass, Gazzaniga, \& Premack, 1973). 


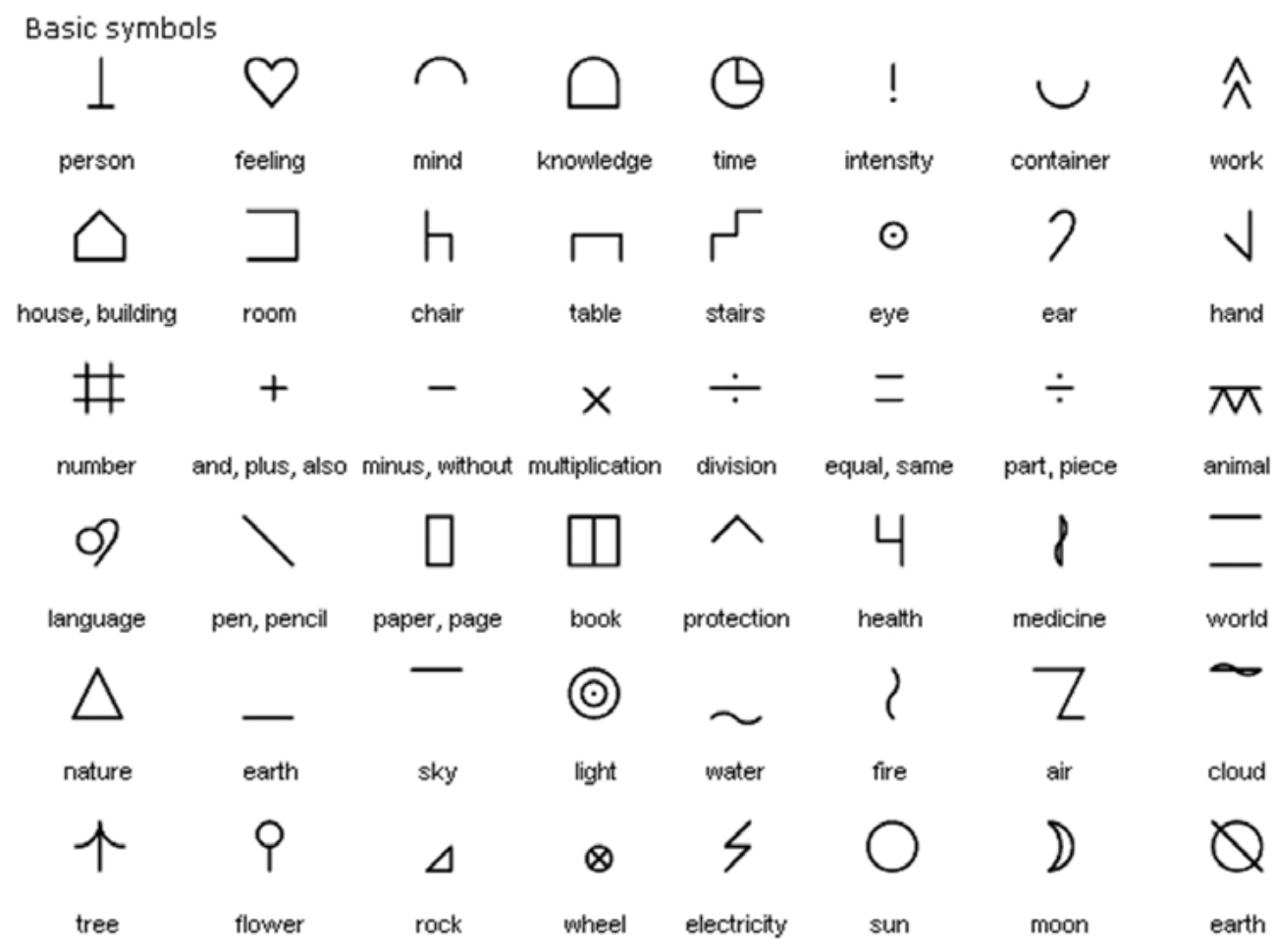

In a training session, 4 globally aphasic patients (U.W., K.M., B.P. \& F.L.) were treated conventionally twice a week for 6 months. None of the patients were considered demented or had an IQ of lower than 80. None of the patients showed significant improvement of expressive speech during conventional therapy except during the first 3 months following acute pathology.

Table 1. Language Assessment of Four Globally Aphasic Patients with the Aachen Aphasia Test

\begin{tabular}{lcccr}
\hline & U.W. & K.M. & B.P. & F.L. \\
\hline Repetition & 29 & 22 & 21 & 6 \\
Naming & 15 & 37 & 22 & 6 \\
Written language & 19 & 19 & 12 & 19 \\
Comprehension & 38 & 24 & 21 & 9 \\
Token Test & 19 & 51 & 51 & 30 \\
\hline
\end{tabular}

UW was a 41-year-old housewife and mother with ischemic infarction. She had right hemiplegia and right hemineglect. She had global aphasia, and buccofacial articulatory and ideomotor apraxia were found. She was unable to name objects and. UW's spontaneous 
speech was extremely non-fluent and contained automatisms. UW could not name objects. UW could comprehend single words and simple commands, but made frequent errors with complex commands.

KM was a 47-year-old master house painter with ischermic infarction. KM's spontaneous speech was also non-fluent and contained automatisms. KM could name $30 \%$ of common objects correctly (one word names). KM had almost no written language or language comprehension.

BP was 55-year-old tailor who suffered a complete left medial cerebral artery infarction. Right hemiparesis and global aphasia were found. BP's spontaneous speech was nonfluent and contained automatisms. BP could repeat one syllable words correctly, but did not freely generate speech. BP's written language was massively disturbed. BP understood single words and simple commands.

FL was a 57-year-old skilled laborer with ischemic infarction. FL had right hemiplegia, right sensory loss, and global aphasia. FL's spontaneous speech consisted of fluent recurring utterances. FL's attempts at naming and repetition tasks resulted only in recurring utterances. FL had extremely poor written language and language comprehension.

Their training method with Blissymbols was as follows: All patients received individual "speech" therapy using Blissymbols at least twice per week for at least 2 months. Symbols were introduced verbally and by simultaneous presentation of pictures or objects or by pantomime of the therapist. The patient's symbol vocabulary was controlled and restrained at the beginning of the session such that new symbols only were introduced after comprehension of originals.

Results of training were as follows: All patients acquired a Blisssymbol lexicon. $3 / 4$ of the patients could produce Blissyntactically correct sentences in response to thematic pictures of questions in Blissymbols. An unexpected finding was the easy articulation of the correct word together with pointing to the corresponding symbol (but these were still one word articulations).

Overall, the tendency to perseverate was milder with the symbols and the patients could overcome their symbol perseveration more easily than their verbal perseveration.

Productivity: The patients displayed capacity for productivity of thought. So while very limited, there is productivity of thought which can be accessed by giving the subjects symbols they can picture or manipulate and, hence, overcome their limited working memories for holding symbols in mind long enough to generate syntactically correct Blisssentences.

\section{Are there other reasons to think that there is a dissociation of language and cognition?}

Yes. Harriet Read (now Cook) studied MR for music cognition and found that MR displays significant music cognition. He showed highly complex music reading abilities, that is, not only the ability to read musical notation out of context, but to read in the context of a score, follow individual parts, and recognise and interpret specific aspects of the score. MR showed a high level of precision in following music as demonstrated by page turning at appropriate points. And as noted above, errors on score reading tasks were close to target.

Although MR had difficulty with word reading, and profoundly impaired performance on comprehending written sentences, he demonstrated no difficulty with recognition of 
musical notation including fairly complex scores. This is particularly interesting because as symbolic systems music and language are organised in very similar ways. Both are highly structured, 'grammatical' rule governed systems.

\section{Contrarians}

Of course, not all researchers find the exact same results. Baldo, et. al. (2005), do find that cognitive performance is diminished with diminished language capacity (from either neural damage or diminished capacity due to shadowing). What is more, they attribute some of the cognitive efficacy of language in cognition to something like "inner speech." Their suggestion is that language facilitates the manipulation of the concepts necessary for solving cognitive tasks. At the same time they do admit that there are a number of cognitive domains, such as visuospatial tasks, that appear to be "unaffected by language impairment" (Baldo, et.al., 2005, 249). Hence, their findings are certainly consistent with the last two sets of findings above.

At the time of their work, Johannsen-Horbach, et.al. (1985) seemed to think that subjects were mapping Blissymbols onto stored linguistic representations. And for one patient who had almost no linguistic competence, the use of Blissymbols did not improve his communicative capacity at all. So, if the Blissymbols are only helping subjects who still have some linguistic abilities, then this study does not demonstrate two independent systems (one linguistic and one cognitive but non-linguistic).

Nevertheless, the symbols used by Glass, Gazzaniga \& Premack and Blissymbols are pictorial. They are clearly not words. They are used in conjunction with words, true. But if they are filling slots in LOT, it might certainly appear that they are «mapping onto linguistic abilities.» It is not clear from their work whether Johannsen-Horbach et. al. even knew of the idea of LOT, in order to consider it when interpreting their results. Since their subjects were using Blissymbols in combination with the few words they retained, it also is not surprising that the one subject who had lost all language would not be able to cobble together words and pictorial symbols in order to communicate. What is more, prior to the training with Blissymbols, none of the four subjects were able to communicate thoughts linguistically, nor to understand sentences. After training they were able to compose word/ symbol combinations into syntactically correct strings. This is just what LOT predicts would be possible if the pictograms are filling slots in the language of thought.

\section{Conclusion}

If Fodor were right and there were a language of thought, and if one's language and cognitive capacities were to dissociate, one would expect it to still be possible for a thinker's cognitive capacities to include productivity, systematicity, recursivity, and the ability to think about actual and non-actual states of affairs. In the research of Varley, et. al., Glass, Gazzaniga, \& Premack (1973) and Johannsen-Horbach et. al. (1985), we find just such capacities among subjects with global aphasia. Their linguistic capacities suffer enormously. They are as Varley says "agrammatical." And yet their cognitive capacities differ little from those had by you or I. This includes, as demonstrated through the research described above, each of 
the four capacities Fodor described. Subjects who learned to use artificial symbols systems developed modest productivity. Also bear in mind that many of these subjects can drive a car, balance their checkbooks, and do other types of activities that require a normal productivity of thought, despite severe agrammaticism.

Varley's subjects in the "communication game," learned to either convey intentions to receivers, or to receive intentions from the senders. This is clearly a form of systematicity of thought.

Varley's (2014) subjects displayed recursivity in mathematical reasoning ability. And MR's musical ability also displayed some of the same kind of cognitive ability.

What is more, those who learned to use Premack's or Bliss's symbols would be able to put together sentences about things present or past, future or false (if they wanted to).

Taken as a whole the research of Varley and her collaborators (and that of the other researchers described here) offers significant evidence of a dissociation between language competence and cognitive competence. Subjects with global aphasia have the latter but lack the former. Therefore, this research offers significant support for the view that there is a language like representational system in the brain that is not a natural language, but more likely a language of thought.

\section{REFERENCES}

Adams, F. (2001). Empathy, neural imaging, and the theory versus simulation debate, Mind \& Language, 16, 368-392.

Adams, F. \& Fuller, G. (1992). Names, contents, and causes, Mind \& Language, 7, $205-221$.

Adams, F., Fuller, G., \& Stecker, R. (1993). Thoughts without objects, Mind \& Language, 8, 90-104.

Baldo, J.V., Dronkers, N.F., Wilkins, D., Ludy, C., Raskin, P. \& Kim, J. (2005). Is Problem solving dependent on language? Brain and Language, 92, 240-250.

Baron-Cohen, L. (1995). Mindblindness. Cambridge, MA: MIT/Bradford.

Baron-Cohen, Leslie, A. \& Frith, U. (1985). Does the autistic child have theory of mind? Cognition, 21, 3746.

Baillargeon, R., Scott, R. \& He, Z. (2010). False-belief understanding in infants, Trends in Cognitive Science, $14(3), 110-118$.

Benn, Y., Zheng, Y., Siegal, M., Wilkinson. I.D., Varley, R., (2012). Language in calculation: a core mechanism? Neuropsychologia 50 (1), 1-10.

Benn, Y., Wilkinson, I.D., Zheng, Y., Cohen Kadosh, K., Romanowski, C.A., Siegal, M., Varley, R. (2013). Differentiating core and co-opted mechanisms in calculation: the neuroimaging of calculation in aphasia. Brain \& Cognition 82 (3), 254-264.

Bliss, C.K. (1965). Semantography (Blissymbolics). Sydney: Semantography (Blissymbols) publications.

Fodor, J.A. (1975). The language of thought. New York: Thomas Y. Crowell Co. Inc.

Fodor, J.A. (1987). Psychosemantics. Cambridge, M.A.: MIT/Bradford.

Fodor, J.A. (2008). LOT 2: The language of thought revisited. Oxford: The Clarendon Press.

Garrett, K. L. \& Beukelmann, D. R. (1995). Changes in the interaction patterns of an individual with severe aphasia given three types of partner support, Clinical Aphasiology, 23, 237-251.

Goldman, A. I. (2012). Theory of mind. In E. Margolis, R. Samuels, \& S. Stich, (eds.) Oxford handbook of philosophy and cognitive science, Oxford: Oxford University Press, 402-424.

Glass, A.V., Gazzaninga, M., \& Premack, D. (1973). Artificial language training in global aphasics, Neuropsychologica, 11, 95-103. 
Hauser, M., Chomsky, N. \& Fitch, T. (2002). The faculty of language: What is it, who has it, and how did it evolve? Science, 298, 1569-1579.

Johannsen-Horback, H., Celga, B., Mager, U., Schempp, B. \& Wallesch, C-W. (1985). Treatment of chronic global aphasia with a nonverbal communication system, Brain and Language, 24, 74-82.

Pinker, S. \& Jackendorf, R. (2005). The faculty of language: What's special about it? Cognition, 95, 201-236.

Siegal, M. \& Varley, R. (2006). Aphasia, language, and theory of mind, Social Neuroscience, 1, 167-174.

Siegal, M, Varley, R., \& Want, S. (2001). Mind over grammar: reasoning in aphasia and development, Trends in Cognitive Science, 5, 296-301.

Varley, R. (2014). Reason without much language, Language Sciences, 46, 232-244.

Varley, R. \& Siegal, M. (2000). Evidence for cognition without grammar from causal reasoning and 'theory of mind' in an agrammatic aphasic patient, Current Biology, 10, 723-726.

Varley, R., Klessinger, N., Romanowski, C., \& Siegal, M. (2005). Agrammatic but numerate, Proceedings of the National Academy of Sciences, USA. 102, 3519-3524.

Willems, R. \& Varley, R. (2010). Neural insights into the relation between language and communication, Frontiers in Human Neuroscience, 4, 1-8.

Willems, R., Benn, Y., Hagoort, P., Toni, I., \& Varley, R. (2011). Communicating without a functioning language system: Implications for the role of language in mentalizing, Neuropsychologia, 49, 3930-3135.

Zimmerer, V., Cowell, P. \& Varley, R. (2010). Individual behavior in the learning of artificial grammar, Memory \& Cognition, 39, 491-502.

Zimmerer, V., Cowell, P.E., \& Varley, R. (2014). Artificial grammar learning in individuals with severe aphasia, Neuropsychologia, 53, 25-38.

Zimmerer, V. \& Varley, R. (2015). A case of “order insensitivity"? Natural and artificial language processing in a man with primary progressive aphasia, Cortex, 65, 212-219.

Fred Adams is Professor of Linguistics \& Cognitive Science and he is Professor of Philosophy at University of Delaware, USA. He has over 150 publications in philosophy and cognitive science.

AdDREss: University of Delaware, Newark, Delaware, 19716 USA. Email: fa@udel.edu 